\title{
La satisfacción de los periodistas de Ecuador, Chile y México frente a la formación universitaria y sus implicancias en el ejercicio profesional
}

\section{Ecuador's, Chile's and Mexico's journalists' satisfaction in relation to university education and its implications in professional practice}

\author{
Fernando Gutiérrez Atala, Javier Odriozola Chéné, Juan José Domínguez \\ Panamá 1 \\ Recibido el 30 de setiembre de 2016 - Aceptado el 2 de noviembre de 2016
}

\begin{abstract}
RESUMEN: En el contexto de una sociedad de la información y el conocimiento, en la que el desarrollo tecnológico avanza a pasos gigantescos, sondear la sensación de suficiencia en los egresados activos en el ámbito laboral es una necesidad imperativa de cara al perfeccionamiento de la profesión y su enseñanza. El objetivo de este estudio es determinar la percepción de los periodistas sobre la formación universitaria y el nivel de satisfacción de éstos con su formación universitaria, incluyendo aspectos de manejo técnico, teórico y práctico. Para ello, se realizaron 120 entrevistas semi-estructuradas a periodistas en activo chilenos, ecuatorianos y mexicanos de los cuatro soportes periodísticos (prensa, televisión, radio e internet). Los resultados muestran que si bien la importancia concedida a la formación académica en el desarrollo profesional varía según el país, los periodistas de cada país perciben una serie de falencias en sus sistemas educativos particulares que hacen necesaria una reformulación de las carreras de Comunicación Social y Periodismo.
\end{abstract}

Palabras Clave: periodismo, práctica profesional, factores de influencia, educación, América Latina ABSTRACT: In the context of an information and knowledge society in which technological development is moving ahead at a staggering speed, probing the sense of sufficiency in the graduates active in the field of work is presented as an imperative need for the profession's improvement and

1 Fernando Gutiérrez Atala es Doctor en Ciencias de la Información por la Universidad Complutense de Madrid y periodista. Jefe y académico del Magíster en Comunicación Creativa de la Universidad Católica de la Santísima Concepción (Chile). fgutierrez@ucsc.cl, http://orcid.org/0000-0003-2289-9224

Javier Odriozola Chéné es Doctor en Periodismo por la Universidad del País Vasco, Profesor titular y Director de la Maestría de Investigación en Comunicación Digital de la Universidad de Los Hemisferios (Ecuador). javiero@ uhemisferios.edu.ec, http://orcid.org/0000-0002-8595-1950

Juan José Domínguez Panamá es Licenciado en Comunicación y Maestro en Periodismo en la Universidad Veracruzana (México). Es investigador y doctorando en Estudios Socioculturales en la Universidad Autónoma de Aguascalientes. josedominguez@uv.mx, http://orcid.org/0000-0003-4268-9099 
its teaching. The objective of this study is to determine the journalistic perception about university education and the satisfaction level of journalists with their university education, including aspects of technical, theoretical and practical management. To meet this aim, forty semi-structured interviews have been conducted with active Chilean, Ecuadorian and Mexican journalists, from the four media (press, television, radio and internet). The findings show that although the importance given to academic training in professional development varies according to the country, Chilean, Ecuadorian and Mexican journalists perceive a series of shortcomings in their educational systems, which needs a reframing of Communication and Journalism careers.

Keywords: journalism, professional practice, influence factors, education, Latin America

\section{Introducción}

En julio de 2014 se creó el grupo de investigación ERP (Estudio de Rutinas Periodísticas), compuesto por investigadores de Chile, Argentina, Ecuador, Colombia y México. Nació para estudiar permanentemente las transformaciones que registran los mecanismos utilizados por los periodistas latinoamericanos en el proceso de recolección, selección y publicación de información, las influencias que podrían afectar a dicho proceso y los efectos que aquello tiene en el producto final que se ofrece al público.

ERP es un estudio de tipo cualitativo con un enfoque descriptivo/exploratorio. El primer informe, en plena etapa de análisis de la información recogida, estuvo orientado a la identificación de las condicionantes que los propios periodistas reconocen al autoevaluar sus rutinas de trabajo. Se elaboró sobre la base de 200 entrevistas semiestructuradas aplicadas a informadores de Buenos Aires (Argentina), Concepción (Chile), Bucaramanga (Colombia), Quito (Ecuador) y Veracruz (México), representantes de cuatro soportes di- ferenciados: prensa escrita, radio, televisión y cibermedios. La segunda etapa se centra en el análisis comparativo de esos condicionantes (Gutiérrez, Odriozola, Aguirre, et al., 2015; Gutiérrez, Ferreira \& Pajoni, 2015; Gutiérrez Atala, Odriozola, Ferreira, Anaya \& Pajoni, 2016).

El equipo ERP lo componen más de 10 académicos de las Universidades: Católica de la Santísima Concepción (www.ucsc.cl), Católica Argentina (www. uca.edu.ar), Autónoma de Bucaramanga (www.unab.edu.co), De Los Hemisferios (www.uhemisferios.edu.ec) y

Veracruzana (www.uv.mx).

La idea de fondo de este artículo, que se enmarca en los resultados del mencionado primer informe, es determinar el peso específico de los condicionantes formativos en los periodistas latinoamericanos para obtener una radiografía de la formación universitaria en términos de satisfacción. En el contexto de una sociedad de la información y el conocimiento en la que el desarrollo tecnológico avanza a pasos gigantescos, sondear la sensación de suficiencia en los egresados activos en el ámbito labo- 
ral se presenta como una necesidad imperativa de cara al perfeccionamiento de la profesión y su enseñanza. En este documento específico, se considera la participación de 120 profesionales de Ecuador, Chile y México, 40 de cada país.

Para lo anterior, se establecieron dos objetivos fundamentales: primero, determinar la percepción sobre la formación universitaria (según los periodistas entrevistados, esta percepción ces homogénea en los países analizados o nos encontramos ante diferentes realidades nacionales?). Y, en segundo lugar, identificar a la luz de lo expresado por los sujetos requeridos en el estudio, el nivel de satisfacción de los periodistas con su formación universitaria, incluyendo aspectos de manejo técnico, teórico y práctico.

\section{Marco teórico}

\subsection{Influencias en el desempeño de la profesión periodística}

El periodismo como profesión no ha estado exento de los cambios surgidos en la sociedad actual y la vorágine que impone nuevas formas de vivir, de comunicarse y de informar. De esta manera, se desarrollan comportamientos comunes entre sociedades con un desarrollo económico, cultural y político similar (Inglehart \& Carballo, 1997, p. 35). Sin embargo, si bien pueden presentar características comunes, también presentan otras derivadas de la propia reali- dad nacional. Esta cuestión también es aplicable al ejercicio del periodismo y en las modificaciones que presenta su influencia en la comunidad.

El estudio de la figura del periodista a nivel latinoamericano involucra el desafío de homogeneizar grupos culturalmente muy diversos. Así, "para estudiar el periodismo como parte de los procesos comunicacionales de América Latina, se hace necesario referirse a los conocimientos tradicionales, la memoria, la investigación, la innovación, las imposiciones económicas, la educación, la política y el diálogo de saberes" (Oller-Alonso, 2016, p. 226).

En tanto profesionales, los periodistas deben ser considerados como sujetos que trabajan dentro de un medio de comunicación que se enmarca en un contexto social específico. Meyer (2004) ofrece una serie de reflexiones orientadas a plantear y profundizar en lo que denomina un "modelo de influencia" de los medios de comunicación (específicamente los periódicos), basado en la premisa de que el principal producto del medio no es la noticia o la información que publica, sino la influencia, principalmente la influencia social, que no está a la venta, pero que podría terminar realzando la influencia comercial. El asunto establece una interesante conexión entre el aspecto informativo y el empresarial, sobre todo en tiempos en que la industria mediática atraviesa momentos difíciles. A su juicio, uno de los principales desafíos de la prensa está en "mantener vivo el periodismo" du- 
rante tiempo suficiente para que los empresarios exitosos de los medios de comunicación del futuro encuentren una forma de captar y vender la influencia que los medios de comunicación están abandonando (2004, pp. 5557). Su propuesta se enfoca a defender la idea tradicional del periodista como guardián de una sociedad libre y su propósito es enfocar sus esfuerzos en la vigilancia del entorno, sobre todo en una época de sobrecarga de información. Este enfoque sería necesario debido a la transición de una sociedad donde la información escaseaba a uno donde existe en exceso, con el riesgo de extraviar a la sociedad y su opinión. A su juicio, la atención pública conforma un nuevo bien, muy escaso y apetecido.

Un estudio dirigido por Hanitzsch (2010) plantea que los condicionantes que afectan al desarrollo de la práctica periodística se producen a diferentes niveles. Clasifica estas influencias en seis dimensiones: a) políticas, tanto de representantes políticos como de lobbys que se desenvuelven en el campo político; b) económicas, relacionadas con la necesidad de la publicidad como forma de financiar las empresas periodísticas; c) organizacionales, relacionadas con los procesos de toma de decisión en los medios de comunicación; d) de procedimiento, en relación a las limitaciones de tiempo y espacio con que trabajan los periodistas; e) profesionales, cuando se habla de las prácticas de trabajo asentadas de acuerdo a convenciones culturales de la profesión, y f) grupos de referencia, que determinan el trabajo del periodista y que están compuestos tanto por la audiencia como por otros actores diversos como compañeros de profesión, amigos o familiares (pp. 11-12).

Por su parte, Oller y Meier (2012) plantean una categorización de las influencias en la práctica periodística en tres niveles: (a) el primer nivel sería el de sistema, entendido como el marco social en el que se desenvuelven los medios de comunicación, relacionándose con otros sistemas de la sociedad; (b) el segundo nivel se focaliza en las influencias de la propia institución mediática, en su estructura y procedimientos habituales; y (c) el tercer nivel hace referencia al periodista como actor que se relaciona con las propias rutinas de trabajo que el periodista desarrolla a nivel individual dentro del medio de comunicación.

En tanto, para Shoemaker y Reese (2014) existirían cinco niveles de influencia: (a) individuales, en relación al propio periodista como creador de los relatos periodísticos; (b) rutinas periodísticas, asentadas en la profesión y, por tanto, tácitamente validadas; (c) organizacionales, de acuerdo a los responsables de toma de decisiones en los propios medios de comunicación; (d) instituciones sociales, donde se encuadran anunciantes, audiencias o grupos de interés; y (e) el sistema, entendido como el sistema social o las ideologías de las sociedades (pp. 8-9).

Es importante destacar que las influencias no se desarrollan horizontalmente 
sino que se establecen siguiendo una jerarquía "que propone importantes distinciones entre los niveles de análisis y sitúa al periodista individual en una red de limitaciones organizacionales e ideológicas" (Reese, 1999, p. 48). Así, los condicionantes ideológicos de los periodistas se ven limitados por condicionantes institucionales, relacionados con la organización jerárquica de los medios y las rutinas periodísticas, que a su vez dependen del contexto social en el que los profesionales de la información y los medios se desenvuelven.

\subsection{La formación académica como parte de las influencias individuales del periodista}

Uno de los factores que constituyen la ideología del periodista es la formación académica recibida. $\mathrm{Al}$ abordar este tema, es habitual el debate entre aquellos que abogan por mallas curriculares focalizadas en la investigación y el pensamiento crítico y los que quieren programas con un gran componente técnico y orientado a la adquisición de habilidades (Shoemaker \& Reese, 1996, p. 72). Sin embargo, para Miguel Wiñazki, director de Capacitación del diario Clarín, "la tarea de informar requiere de un saber diferenciado, teórico y práctico: la reflexión filosófica sin el oficio cotidiano de informar suele estar vacía, y el oficio sin la reflexión es ciego, y suele desviarse hacia un operativismo superficial" (Foro de Periodismo Argentino, 2008, p. 208).
En el contexto latinoamericano, en el año 2008, la Federación Latinoamericana de Facultades de Comunicación Social identificó 1.742 centros de enseñanza universitaria en el que se ofertaban programas de comunicación y periodismo, estando distribuidos de la siguiente manera: 67 en Centroamérica y Caribe (Costa Rica, Cuba, El Salvador, Guatemala, Honduras, Nicaragua y República Dominicana); 1006 en México; 193 en la región Países Andinos (Bolivia, Colombia, Ecuador, Perú y Venezuela); 115 en la región Cono Sur (Argentina, Chile, Uruguay y Paraguay) y 361 en Brasil (Felafacs, 2009, p. 11).

Los planes desarrollados en las facultades latinoamericanas responden a tres modelos: culturalista, profesionalista y comunicólogo. "El modelo humanista o culturalista considera que el mejor comunicador es el más culto, por lo que incide en cursos de ciencias sociales y humanas; por su parte, el modelo práctico profesional pone el acento en los aspectos de la práctica, en sintonía con las recomendaciones de la Sociedad Interamericana de Prensa, mientras el modelo comunicacional entiende el periodismo como una de las variantes de la comunicación, con la pretensión de formar profesionales preparados en sus diversos aspectos y luego fijar esfuerzos en una especialidad determinada" (López, 2010, p. 233-234). Las críticas a las facultades latinoamericanas formadoras de periodistas y comunicadores se han centrado "en su falta de definición académica y la ambigüedad de sus 
perfiles; su escasa vinculación con la sociedad; su incapacidad de conjugar su proyecto educativo con las salidas profesionales de sus egresados, así como su limitada capacidad productiva (Mellado, 2010, p. 17).

En el contexto específico de los países considerados en este estudio, puede señalarse que en Ecuador, la radiografía de las universidades con grados en Comunicación Social o Periodismo muestra en primer lugar un entorno en el que los académicos se centran prácticamente en la labor docente, obedeciendo a la estructura universitaria general del país que va modificándose lentamente; luego unos planes académicos con acercamiento a la parte práctica y técnica (Punín, 2012, p. 20); y finalmente la inexistencia de una malla curricular que implemente al periodismo digital y cubra sus exigencias e infinidad de aplicaciones (Punín, Rivera \& Cuenca, 2014, p. 44).

En Chile, desde la creación de la primera escuela de Periodismo en la década de 1950, los programas abastecedores de profesionales mantuvieron un ritmo de crecimiento estable hasta la década de los 80 y 90, donde se registró una explosión en la matrícula. Actualmente, no existe un perfil claro respecto de lo que se entenderá por "periodista", pues el título y grado que entregan las universidades lo habilita para las funciones informativas propiamente tal, pero también para otra amplia gama de acciones que van desde las relaciones públicas hasta la gestión estratégica de la comunicación (Mellado, 2012, pp. 382-399).

En México para entender el proceso de formación de profesionales en comunicación y periodismo, es necesario comprender su contexto, pues en un principio, para la década de los 60, surgieron las escuelas de Periodismo, de manera tardía en comparación con el resto de Latinoamérica. Sólo tres instituciones aparecieron para formar periodistas profesionales. Para las siguientes tres décadas el número se elevó a los varios cientos de instituciones, con una gama de opciones en la formación de profesionales mismas que hoy en día se centran en la comunicación social, corporativa, multimedia y periodismo (Hernández, 2004).

Sin embargo, es importante señalar que el tema sobre la enseñanza viene inmerso en un debate sobre el campo y el objeto de estudio mismo de la comunicación, donde -entre tantos autores y académicos que han abordado el tema- Raúl Fuentes Navarro (2014) y María Elena Hernández (2004) han hecho importantes aportes en el sentido de distinguir en la enseñanza de la comunicación una amplia variedad de opciones que integran las escuelas de comunicación en un estándar profesionalizante y tecnificador, como formadores de profesionales conocedores de saberes y dominadores de las herramientas tecnológicas en el oficio de la información, es decir, sin establecerlo, la base de la formación de los distintos perfiles de comunicación en México 
tienen como pilar fundamental el periodismo.

Por lo tanto, se plantea la necesidad de crear un eje formativo que, además de adaptable, abarque todas las aristas posibles de un entramado del tamaño de la comunicación misma. Sin embargo, la guía está grabada en la experiencia de los mismos periodistas, que deben ser escuchados para este objetivo. Este escenario genera una necesidad de adaptación: "los vertiginosos cambios actuales, que nos atrevemos a calificar por su velocidad y envergadura de exponenciales, naturalmente nos obligan a todos a una forzada y continua necesidad de adaptación" (Seijas, 2001, p.199). Una modificación transversal, que se desarrolle en función del cambio constante que caracteriza el entorno laboral informativo, y que aporte soluciones reales que ayuden a consolidar la profesión.

En este sentido, dotar al periodista de herramientas pertinentes que potencien su capacidad de influencia -por ejemplo aumento en el poder de negociación informativa, potenciamiento del proceso formativo o asumir la labor del profesional de la información como una tarea de mediación público-realidad informable- apuntan a otorgar al periodista el nivel suficiente de autonomía profesional, que le permita el correcto desempeño de sus labores informativas (Gutiérrez, 2012, pp.36-40) y que lo lleven a enfrentar adecuadamente los cambios sociales, culturales y profesionales que generan las innovaciones y la reinvención permanente y continua del periodismo, con la incorporación de nuevas reglas y nuevos valores dentro del debate sobre la calidad (Deuze, 2004, pp.139-152 y 2005, pp. 442464.). Esta exigencia está directamente relacionada con valores vinculados a la ética profesional, como la responsabilidad, la credibilidad y la independencia.

Como se ha planteado, el periodismo se está convirtiendo en una profesión cambiante. Fuller (1997) adelantó que el nuevo medio interactivo amenaza el statu quo y promete una excitante forma nueva de aprender sobre el mundo. Por su parte, Kovach y Rosenstiel (2001) presentan un argumento irrefutable que demuestra que el negocio noticioso está pasando por un momento de transición. De acuerdo con los autores, cada vez que se presentó un periodo de significativo cambio social, económico y tecnológico, ocurrió una transformación en las noticias. Por estos días el cambio en las noticias puede ser más dramático, pues por primera vez en nuestra historia, cada vez más la noticia es producida fuera del periodismo y argumentan que la nueva tecnología, junto con la globalización y la conglomeración de medios, está causando un alejamiento de un periodismo que está conectado a la construcción de ciudadanía y que apoya a una democracia saludable. El periodismo enfrenta entonces un proceso de redefinición, ajustándose a las fuerzas perturbadoras que lo rodean. Así, no es una sorpresa que las discusiones sobre los foros de periodismo participativo se centren con frecuencia en debates defensivos sobre 
qué es el periodismo y quiénes pueden llamarse legítimamente periodistas.

Así, todo parece indicar que la cualificación profesional sería la herramienta más eficaz para ejercer control frente a las fuentes, desde el punto de vista de saber quiénes son, cuáles son sus intereses y sus estrategias de persuasión. Por eso, la premisa sería advertir esta situación a los informadores, previniéndolos desde su formación en la universidad, ya que -a juicio de Elías (2004)- el estudio profundo de la identificación y la idiosincrasia de las fuentes en cada área de especialización, así como de investigaciones realizadas en facultades que demuestren cómo se comporta cada tipo de fuente, debería ser el eje fundamental de la docencia en ciencias de la información si queremos paliar esa vulnerabilidad del periodista.

\subsection{Estructura mediática}

Si bien cada país tiene sus particularidades en su relación medios-sociedad, el problema de los medios existe en todas las sociedades (McChesney, 2004, p. 16). Un periodista no consiste en un autómata transmisor de contenidos, por el contrario, actúa en función de valores y paradigmas y esta materia subjetiva deja adherencias en sus crónicas, aun cuando se esfuerce en ser imparcial, un invisible mensajero de la actualidad.

Sin importar la época en la que se encuentre, el periodista desarrolla su tra- bajo acorde a su propia realidad. Especialmente en el caso chileno, después del periodo de dictadura, y en comparación a otros países, se instala una dinámica poder-medios en que, aún en democracia, "existe la misma tensión entre aquellos que tienen el poder y los que no, la batalla solo asume diferentes formas. Los medios están en el centro de los conflictos por poder y control en cualquier sociedad" (McChesney, 2004, p. 17).

Trascendiendo las décadas, a nivel latinoamericano es común que "aquellos en el poder generen un sistema de medios que apoya su dominación y minimiza posibilidad de oposición efectiva". Todo esto se basa en la idea de que el uso de los medios de comunicación como principal vehículo para hacer política y tomar al asalto los gobiernos "ya es una realidad en los países de economía. La casuística es variada, aunque similar en lo esencial" (Serrano, 2012, p. 22).

Este escenario de competencia entre conglomerados periodísticos ha llevado la batalla a todos los soportes informativos, desde impresos a digitales. Por lo que el aspirante a periodista de hoy, se enfrenta a un cuadro de muchas partes que cambian a una velocidad vertiginosa.

\subsection{Influencia de la tecnología}

La presión que ejerce el desarrollo tecnológico conlleva a una serie de nue- 
vos desafíos para el profesional de las comunicaciones. No solo la necesidad de formación constante para evitar caer en la obsolescencia, sino también una larga lista de vicios que se desprenden de la misma tecnología, como la excesiva comodidad, el reporteo de oficina, entre otros. Si bien el periodista tiene amplias facultades, en último extremo, se subordina a la voluntad editorial de la firma que apoya el medio de comunicación en el que trabaja y en las que -a veces- conduce a que algunos profesionales deban introducirse en ámbitos informativos de dudosa calidad" (Reig, 2007, p. 89).

Este contexto socio-cultural que se puso en marcha en el siglo XX, sumado a la modernización de los procesos permite afirmar que en América Latina "la revolución tecnológica, la convergencia mediática y el papel del consumidor han transformado los ritmos y los modos en que se producen los mensajes, y con ello, las formas de expresión del periodismo" (Mellado, 2009, p. 197).

Es la tecnología la que marca, en muchos casos, los ciclos evolutivos de determinados avances y la que acorta la duración de los mismos cada vez a mayor velocidad (Canga, 2001, p. 34). Todo decanta en las redes sociales, plataformas que permiten un interminable flujo de información de alta valoración social y comunitaria, pero que no necesariamente es originada por profesionales de las comunicaciones, lo que actualiza y da sentido a la idea de una "mayor democratización de la información” (Ramonet, 2011, p. 23), pero al mismo tiempo da el punto de partida para una serie de riesgos y desafíos de actualización y re-perfilamiento a los profesionales de la información.

Desde toda perspectiva, la tecnología propició -y seguirá propiciando- un cambio en el sistema de comunicación, desde el momento en que introdujo nuevas formas de creación colectiva de contenidos" (Alonso, 2010, p. 24).

En referencia a la sobreabundancia de datos, una de las primeras razones que exista este caudal de información es por "las nuevas tecnologías de la información y la comunicación, porque aceleran el ritmo de la vida, la velocidad de la transmisión y las posibilidades de compartir información". (Servicio Observatorio Internet, 2001, p. 30). A este caudal, también se le ha llamado "asfixia comunicacional", que es definida como "una sobreabundancia de información que degenera en la supresión de la libertad" (Servicio Observatorio Internet, 2001, p. 70).

\subsection{Marco deontológico}

La presión que ejerce el desarrollo tecnológico conlleva a una serie de nuevos desafíos para el profesional de las comunicaciones. No solo la necesidad de formación constante para evitar caer en la obsolescencia, sino también una larga lista de vicios que se desprenden de la 
misma tecnología, como la excesiva comodidad, el reporteo de oficina, entre otros.

Cuando el periodismo se encuentra en estas circunstancias, la deontología corre el riesgo de tornarse meramente decorativa. Si bien el periodista tiene amplias facultades, en último extremo, se subordina a la voluntad editorial de la firma que apoya el medio de comunicación en el que trabaja y en las que -a veces- conduce a que algunos profesionales deban introducirse en ámbitos informativos de dudosa calidad" (Reig, 2007, p. 89).

\section{Metodología}

La herramienta metodológica utilizada para esta ponencia fue la entrevista semiestructurada y se centró en extraer resultados desde dos perspectivas. En primer lugar, por medio de una entrevista de respuesta espontánea, se indagó en la determinación de la presencia de diez condicionantes pre definidas que marcan la labor periodística, que fueron: ideológicas, económicas, políticas, laborales, tecnológicas, de presión social, de rutinas profesionales, jerárquicas, de condiciones de trabajo y formativas. Sin embargo, para este caso se han considerado sólo las condicionantes de corte formativo. En segundo lugar, se realizó un análisis mediante respuesta sugerida, que permitió profundizar en los aspectos concretos planteados por los entrevistados.
Para ello, se realizaron 120 entrevistas en profundidad en total, a periodistas en activo en cada uno de los subgrupos nacionales (40 por país y 10 en cada soporte: prensa escrita, radio, televisión, soportes digitales). Luego, las respuestas fueron transcritas y ordenadas en tablas sometidas a análisis por los investigadores mediante, hasta llevarlo al punto de saturación (Báez y Pérez de Tudela, 2009, p.95) que delimitó los resultados que se exponen a continuación.

\section{Análisis}

\subsection{Análisis cuantitativo}

$\mathrm{Al}$ analizar el peso de los condicionantes relacionados con la formación recibida, puede observarse que la importancia otorgada por los periodistas ecuatorianos, chilenos y mexicanos varía. En la tabla 1 se constata que los periodistas ecuatorianos entrevistados, al ser preguntados por los condicionantes que reconocen que afectan al desarrollo de un periodismo de mejor calidad en su rutina laboral, mencionan en un $17,5 \%$ de las ocasiones los condicionantes formativos. En el caso de los periodistas chilenos el porcentaje es mucho mayor siendo mencionado por un $72,5 \%$ de los entrevistados. Finalmente, en el caso de México, en coincidencia con Ecuador, dicho condicionante representa solo un $17,5 \%$ del total del estudio hecho en este país.

Sin embargo, la metodología de este proyecto no se limita a medir la presen- 
cia de los diferentes condicionantes en la pregunta planteada a los entrevistados, sino que profundiza, en una segunda fase de respuesta sugerida, en cada uno de los condicionantes. Gracias a esta profundización, se puede ampliar el análisis y categorización de cada uno de estos condicionantes, también de los condicionantes relacionados con la formación académica.

Tabla 1. Presencia de los condicionantes formativos

\begin{tabular}{|c|c|c|}
\hline País & $\begin{array}{c}\text { Influencia de los } \\
\text { condicionantes } \\
\text { formativos }\end{array}$ & Porcentaje \\
\hline Ecuador $(n=40)$ & 7 & $17,5 \%$ \\
\hline Chile $(n=40)$ & 29 & $72,5 \%$ \\
\hline México $(n=40)$ & 7 & $17,5 \%$ \\
\hline Total $(n=120)$ & & \\
\hline
\end{tabular}

Fuente: Elaboración propia

\subsection{Análisis cualitativo}

Para organizar la información recogida se presentan los resultados de acuerdo con cinco ejes temáticos que organizan la opinión de los periodistas entrevistados: importancia de la formación universitaria, aporte profesional, falencias, causas de la brecha teoría-práctica y percepción general.

\section{a) La importancia de la formación uni- versitaria}

Los periodistas ecuatorianos reconocen la importancia de la formación universitaria. Este hecho se ha visto reforzado, desde el año 2013, por la exigencia plasmada en el Artículo 42 de la Ley Orgánica de Comunicación de que "las actividades periodísticas de carácter permanente realizadas en los medios de comunicación, en cualquier nivel o cargo, deberán ser desempeñadas por profesionales en periodismo o comunicación" (Asamblea Nacional, 2013, p. 9). Los propios periodistas son conscientes de esta situación: "la ley te obliga ahora que todos tienen que tener tercer nivel, es decir, la licenciatura, haber acabado la carrera de comunicación" (Sujeto 152). Así, en la actualidad realmente "es una responsabilidad muy grande, no solo de la empresa sino del mismo periodista en tener su título profesional para que su trabajo sea respetado y el mismo esté fuera de cualquier riesgo de cumplir un trabajo que significa informar de un hecho si es que no tiene su título profesional de periodista» (Sujeto 142).

En los periodistas chilenos, las opiniones son variadas. Hay sujetos cuyas críticas a la formación es lapidaria y otros casos en los que la desconexión con la calle es entendida como algo normal 
del proceso formativo, sin dejar de ser una condicionante para la rutina laboral (Sujeto 23). En la misma tónica, las presiones políticas son un escenario recurrente y condicionante en la rutina informativa, sumado a acontecimientos fuertes como cobertura de homicidios. Opiniones más radicales afirman que el estudiante "no pone en práctica nada de lo que enseñan en la universidad" (Sujeto 25). Por otro lado, se menciona la formación universitaria como la proveedora de herramientas básicas para el reporteo también. Por lo tanto, la investigación no demuestra consenso en cuanto a la formación universitaria como un aporte importante para los sujetos de estudio, en el caso Chile.

Resulta interesante observar que, para los periodistas mexicanos, no es cuestionable la importancia de la formación universitaria, la ausencia de la mención hace indicar dicha cuestión. La lectura a las respuestas de los periodistas hace referencia a una ausencia de un compromiso en la actualización y capacitación correspondiente a los empleadores o dueños de medios, el gobierno o un organismo independiente, porque la universidad ya cumplió su papel de formadora. "Por ejemplo la empresa debe comprometerse con la capacitación, después sería bueno contar con un sindicato o bien que hubiera un organismo externo que apoye en este sentido" (Sujeto 207), al tiempo que asumen los periodistas el hecho de que el periodismo por naturaleza requiere de una formación constante en un sentido autónomo: "Creo que cada periodista debe preocuparse por su actualización disciplinaria, pero también sería ideal que cada medio tenga un programa de actualización permanente"(Sujeto 219).

\section{b) Aportes de la formación universita- ria al desarrollo profesional}

Por norma general, los periodistas ecuatorianos reconocen que los conceptos adquiridos en las facultades de comunicación, les ha permitido un mejor desempeño laboral: " todo lo que aprendí en la universidad lo pude plantear en las notas" (Sujeto 121). Esos conocimientos adquiridos se concentran en "todo lo que tiene que ver con redacción, gramática, géneros..." (Sujeto 122). También en la documentación informativa: "Yo al periodismo le debo la herramienta de cómo conseguir la información, la carrera de periodismo me enseñó ciertos pasos, ciertas pautas para yo poder desenvolverme en la recopilación de datos" (Sujeto 125). Se detecta, a su vez, una profundización en "la lectura de discursos, en escenario urbanos, interpretación informativa, en ética..." (Sujeto 144), que permite "asentar las bases para que pienses de una manera crítica" (Sujeto 159) y "entender cómo llegar al público, el cómo llegar a la gente" (Sujeto 147).

La síntesis de las opiniones de los sujetos chilenos entrevistados, se concentra en la siguiente afirmación: "Yo aprendí mi oficio, recibí herramientas valiosas en la universidad, pero el porcentaje más alto lo aprendí en la cancha, en los medios, con otros periodistas" (Sujeto 2). Se destaca 
que en la universidad se enseña el componente técnico: cómo hacer bien una cuña, cómo escribir bien una nota, etc. "pero nunca te enseñan a relacionarte en un ambiente laboral" (Sujeto 11). Los periodistas que salen a trabajar, en su mayoría "llegan mal preparados y con conocimientos que no le sirven de nada en el mundo real. Se enseña demasiado sobre la teoría, pero esos conocimientos en el mundo real no son válidos" (Sujeto 24). En experiencias del mismo sujeto, la academia no te prepara son las encrucijadas éticas y las presiones editoriales.

El periodismo mexicano atraviesa cuestionamientos y juicios por parte de la sociedad, en parte por el empoderamiento de la sociedad mediante un ejercicio de información ciudadana gestionado a través de las redes sociales virtuales, lo cual exige un discurso más crítico a los periodistas. Frente a ello, pocos son los periodistas que reconocen la importancia de una formación universitaria, se enfocan más en la necesidad de una actualización y capacitación constante sobre todo en el ámbito del manejo de las tecnologías, sin embargo, los propios periodistas reconocen que "el periodista de hoy debería tener: lectura de compresión, bagaje cultural amplio, al menos un idioma extranjero a nivel básico, redacción avanzada, lenguaje, lectura crítica de los medios, ética y eso lo brinda estudiar una carrera" (Sujeto 222).

\section{c) Falencias de la formación universitaria}

Los periodistas ecuatorianos consideran que en la enseñanza universitaria "se dan las bases y herramientas, pero creo que en todas las universidades falta el tema práctico, tal vez esté mucho mejor pero el tema práctico es lo que falta en muchas universidades" (Sujeto 157). Así, "los chicos que egresan de la universidad y se topan con ese nuevo escenario, creen saberlo todo al momento en el que salen, pero otra cosa es el lado laboral hay un fuerte choque generacional en ese aspecto (...), porque las Universidades tampoco les han enseñado a investigar" (Sujeto 131). En definitiva, detectan un desfase, "el desencuentro entre el proceso de formación -carrera universitariacon el desarrollo de las actividades que desarrolla un periodista en un medio de comunicación. (Sujeto 148).

En el lado contrario, otros periodistas afirman que existen "periodistas profesionales que se pasaron cuatro años hablando del qué, quién, cómo, cuándo, dónde... y la formación en cultura general sobre economía, política, informática, derechos, etc. es sumamente deficiente" (Sujeto 153). Otros periodistas afirman que salieron de las aulas universitarias "sin saber que significa reportear que es una palabra básica en periodismo, entonces si es un problema, cómo estudiaste cuatro años y medio con propedéutico y sales y no sabes cosas básicas, nunca hiciste una cobertura real. (...) te hablo por mi caso y por los de mi generación que ahora están en medios también" (Sujeto 123). Algunos periodistas consideran que se han forjado como periodistas "en el campo, en el día a día" (Sujeto 129). Así, "el comunicador se hace en el día a día. Los conocimientos 
de la universidad son muy generales, no se compagina la academia con la realidad. El salir de la universidad fue, para mí, salir al mundo sin armas. Los medios son los que te forman" (Sujeto 135).

Por su parte, para los periodistas chile$\underline{\text { nos, }}$ el principal problema reconocido por los periodistas en la base formativa, radica en la diferenciación entre teoría y práctica. Se afirma que "la universidad te da, quizás, una forma genérica de cómo relacionarte con el mundo" (Sujeto 31). Se da en algunos casos que los editores afirman incluso que "los periodistas que salen de la universidad no están capacitados en lo absoluto para poder ejercer su labor de una forma aceptable" (Sujeto 33).

Se establece también como limitante, en algunas casas de estudio, la falta de contenidos relativos a reporteo en televisión y una desconexión con el medio por la falta de pasantías (Sujeto 34). Profundizando en contenidos, los resultados evidencian molestia por parte de los profesionales para con su formación reporteril, especialmente en lo relativo a educación cívica, cargos, lenguaje técnico y detalles de protocolo y otros conocimientos de comunicación en organizaciones (Sujeto 36). Sumado a lo anterior, los sujetos destacan la necesidad del desarrollo de habilidades blandas o un "instinto". Especialmente en televisión, donde se cuenta con la presencia de un camarógrafo, el llegar a las fuentes es especialmente difícil y se afirma que es un tipo de habilidad "que no se aprende sino en la práctica” (Sujeto
37). Complementa este argumento la afirmación de una falta de acercamiento a lo psicológico, el tratamiento de situaciones de dolor o catástrofe (Sujeto 6). En una arista poco frecuente, dentro del grupo de estudio se menciona como un problema el que no se forme a los profesionales con visión de empresa. Si bien las habilidades técnicas y teóricas son efectivas, falta un enfoque de marketing personal. Contrario a eso, según menciona el Sujeto 39, el discurso en la escuela en que se formó era: "Estudia harto, porque trabajo no hay".

El discurso de los periodistas mexicanos camina en torno a la distancia del aula a la realidad del mundo en la vida cotidiana; "Trato de hacer mi trabajo, como a mí me han enseñado tanto en la universidad como en el campo laboral, siempre es un aprendizaje constante- te preparan para el periodismo, pero no para lo que te topas cuando sales de la escuela, te "chamaquean" a cada rato hasta que agarras la onda" (Sujeto 231). Por otro lado, y lo que pareciera como una situación dada por naturaleza del ejercicio, existe una percepción sobre el periodismo basada en el arte del oficio de reportear, donde las habilidades periodísticas no dependen de la formación universitaria, sino de las habilidades y destrezas que cada periodista debe desarrollar en el campo de trabajo; "En cuanto a la capacitación más allá de una formación universitaria, este trabajo es de oficio y el informarse día a día, el entrevistar a diferentes personas y el trabajo arduo, el leer y conocer la agenda nacional e internacional van 
dando una ventaja y experiencia laboral" (Sujeto 225).

\section{d) Causas de la ruptura entre la acade- mia y la profesión}

Al hablar de las causas de esta ruptura entre la formación académica y la práctica periodística, hay periodistas ecuatorianos que consideran que "las universidades dicen cosas que no son tan reales, porque tienen profesores que no han estado en el medio" (Sujeto 159). Otros consideran que el desfase vivido en su transición de la formación académica a la práctica periodística "se asienta en un problema en la malla, porque no existía una visión de la realidad que se necesitaba en el Ecuador. Las mallas estaban desactualizadas" (Sujeto 128). Otros periodistas aducen un problema estructural que provoca un estancamiento en "varios temas especialmente ideológicos dentro de las universidades y del sistema educativo en sî" (Sujeto 160).

Los periodistas chilenos, en casos particulares, mencionan que la formación "era más avocada a gente que se iba a dedicar a docencia u otras áreas de la comunicación, a la investigación, pero no al periodismo de prensa" (Sujeto 28). Eso se debe a que muchos de los profesores "nunca ejercieron, no trabajaron en medios reales" (Sujeto 14). Dentro del grupo de estudio se hace el paralelo entre teoría y práctica y se afirma que se aprende mucho más en tres años de calle que en cinco de academia. Esta brecha era especialmente notoria en mallas antiguas en las que las oportunidades de reporteo y pasantías eran escasas (Sujetos 21 y 27). Todos los días se viven situaciones que no se enseñan en las aulas. El sujeto 5 afirma que el periodista "se hace el 25\% en la academia y el resto en la calle". Y es una conclusión a la que llegan todos los consultados.

Los periodistas mexicanos no expresan como tal una ruptura. Por lo argumentado, pareciera que la formación universitaria es una primera etapa elemental para ejercer el periodismo. Las ideas de los periodistas denotan la necesidad de vinculación entre medio, gobierno y entes externos para una capacitación y actualización constante; "En cuanto a la capacitación, son pocos los cursos que se dan para los periodistas, en una ocasión asistí a uno de la UV, otros de la Comisión de Periodistas" (Sujeto 215). "La empresa periodística y organismos externos deben de proporcionar la capacitación y actualización a los periodistas" (Sujeto 238). En este sentido, el principal aspecto en el cual palidece la universidad es de índole económico, puesto que las opciones de actualización profesional y capacitación que ofertan las universidades son escasas y difíciles de costear. "La actualización que se brinda es de protección a los periodistas a través de cursos que promueve el mismo gobierno por medio de la Comisión de Atención a Periodistas y una que otra vez las empresas, pero no alcanza" (Sujeto 218). "No tengo quien me pague mis cursos, yo los pago y asisto a los que organice la Comisión de Periodistas" (Sujeto 220), "A veces 
también lo que ha organizado la Comisión de Periodistas sobre cómo protegernos, pero en sí, en sí, sobre periodismo muy poco, a nadie le interesamos, esa es la verdad" (Sujeto 236).

\section{e) Percepción de la formación univer- sitaria actual}

Esta visión se ha visto configurada por la propia experiencia vivida por los periodistas durante su formación académica, que, en ocasiones, dista de la situación actual.

Así, algunos periodistas ecuatorianos consideran que la formación ha mejorado en estos últimos años. "En la universidad no teníamos todos los elementos técnicos, no era tan avanzada la enseñanza como ahora veo que han avanzado en la universidad pública donde yo me formé" (Sujeto 145). Los mismos periodistas detectan que hay "una nueva ola de gente que llega de aulas, con mucha más capacidad para poder emitir comunicación y hacer información y noticias responsablemente (...). Antes, no se le daba tanta importancia al periodismo (...) Hoy esta formación que tienen muchos jóvenes es mucho más "criteriosa”, específica, cautelosa e inclusive, esta palabra que tanto repito, responsabilidad, es mucho más responsable en lo que hace, dice, proyecta" (Sujeto 143).

Los periodistas chilenos, debido a un factor lógico de condicionantes rutinarias (tiempo), presentan un distanciamiento de las casas de estudio. En este contexto, su opinión sobre los currículos actuales no es profunda. Pero los sujetos valoran positivamente el que se cubran detalles tales como la empresa informativa, marketing, comunicación organizacional y demás herramientas que mejoren su autonomía y la capacidad de "ser la propia empresa" (Sujeto 39). Se destacó la carencia de formación en el ámbito empresarial, se preparaba mucho para ser la hormiga y no para ser el que está a cargo (Sujeto 7) y, a través de las nuevas mallas curriculares, las mejoras y actualizaciones de contenido son patentes. A lo anteriormente mencionado se le suma la realidad específica de cada escuela de Periodismo y sus recursos. "La carencia de equipos tecnológicos se traduce en una condicionante para los estudiantes al no estar actualizados con las tecnologías estándar del entorno laboral" (Sujeto 29).

En el caso de los periodistas mexicanos, el silencio también habla. No se dice mucho sobre el papel de las universidades en su formación, son escasos los señalamientos entre la imaginación del aula y la realidad fuera de ella. Por ejemplo, el sujeto 231, aunque al parecer ello se debe a una situación ya heredada por costumbre y adjudicada al hecho de egresar de una carrera universitaria sólo con los conocimientos teóricos y completar la formación profesional en la práctica.

Por otro lado, existe la percepción de que los conocimientos brindados en la academia son suficientes para brindar cierta autonomía en el perfil profesional 
del periodista, es decir, ahora corresponde al propio periodista, una vez egresado del aula, hacerse responsable del desarrollo de sus conocimientos y habilidades en el trabajo de reporteo y generación de la información, “...Creo que cada periodista debe preocuparse por su actualización disciplinaria..." (Sujeto 216), "Por voluntad propia tengo la capacitación necesaria para desarrollar mis labores" (Sujeto 224).

\section{Conclusiones}

Los periodistas ecuatorianos muestran su disconformidad con la formación académica recibida; $y$, sin embargo, son conscientes de la importancia de la misma desde la entrada en vigor de la Ley Orgánica de Comunicación. Las principales críticas a las facultades de Comunicación del país, se concentran en la falta de consonancia entre lo aprendido en la universidad y las habilidades posteriormente desarrolladas en el ámbito laboral. Las respuestas sugieren que los periodistas entrevistados, por norma general, achacan esta problemática a un modelo de enseñanza demasiado culturalista que no profundiza ni en las herramientas técnicas habituales en la profesión periodística, ni en otras áreas de la Comunicación que han visto incrementada su importancia en los últimos años. Sin embargo, los profesionales ecuatorianos parecen percibir un cambio en el desarrollo curricular de las carreras de Comunicación Social en Ecuador, en el que, hasta cierto punto, se están incorporando los co- nocimientos técnicos, sin abandonar las bondades del modelo culturalista a la hora de formar periodistas íntegros, reflexivos y conocedores de su entorno social.

En el caso chileno, la condicionante formativa toma el podio con una alta presencia de reconocimiento por parte de los periodistas. Si bien el consenso no es absoluto sobre el aporte de la formación universitaria, existen opiniones con radicales diferencias, desde profesionales que apuntan a que la universidad le otorgó las bases teóricas y técnicas para aplicar en su rutina; así como existen sujetos de estudio que plantearon la total inutilidad de la formación universitaria. Lo que sí es claro, es que - por lo menos en años anteriores- la formación académica carecía de elementos prácticos y tecnológicos suficientes. Contexto que, de entrada, explica el descontento con la formación universitaria.

Del estudio completo y de manera global, la condicionante formativa es de las menos presentes en los resultados de la investigación en México. Las lecturas orientan la interpretación sobre de que el papel de la universidad en su formación de profesionales no es cuestionable, ello tiene que ver en primera instancia por la escasa cultura de formación especializada que se tiene en periodismo, debido al tardío florecimiento de las escuelas de periodismo en México y la abrupta llegada de las escuelas de comunicación y la masificación del número de centros y la diversificación en su formación. 
Por otro lado, corresponde a las universidades en México el asumir una postura de vinculación con el entorno para que la formación profesional de periodistas se complemente con el ejercicio en la práctica durante su formación. Asimismo, la circunstancia apunta a que la universidad puede asumir un papel de gestor para el diseño de una currícula capaz de satisfacer las demandas en la formación de los periodistas actuales la cual puede complementar con la oferta de cursos de capacitación, actualización y especialización profesional que involucre a varios actores entre el gobierno, los propietarios de medios de comunicación, y distintos sectores de la sociedad como los sindicatos y las asociaciones para fortalecer el ejercicio periodístico y de información con un sentido crítico.

En definitiva, si bien el peso otorgado a la importancia de la formación académica en el desarrollo de un periodismo de calidad varía según el país, los pe- riodistas ecuatorianos, chilenos y mexicanos muestran una serie de falencias en sus sistemas educativos que hace necesario una reformulación de las carreras de Comunicación y Periodismo. La responsabilidad de esta cuestión recae en las propias universidades que deben configurar programas de estudios acordes a las necesidades del mundo laboral. Sin embargo, no deben olvidar la formación teórica, pero se debe acompañar al alumno en la puesta en práctica de estos conceptos en consonancia con el entorno tecnológico actual. Para ello, la presencia de la comunicación digital debe ser transversal y no limitarse a un determinado número de asignaturas. Por tanto, deben repensarse las mallas curriculares, de tal manera, que estas se adecúen a las necesidades de la sociedad actual, superando los problemas estructurales e ideológicos, que, tradicionalmente, ha adolecido la formación universitaria en Comunicación y Periodismo en América Latina.

\section{Referencias Bibliográficas}

Alonso, J. (2010). Cibermedios: conceptualización y tipologías. En M.A. Cabrera (ed.) Evolución tecnológica y cibermedios. Comunicación Social: Zamora.

Báez y Pérez de Tudela, J. (2009). Investigación Cualitativa. Madrid: ESIC.

Canga, J. (2001). Periodismo e Internet: nuevo medio, vieja profesión. Estudios sobre el mensaje periodístico, 2001 (7). Recuperado de: http://revistas.ucm.es/index. php/ESMP/article/view/ESMP0101110033A/12804

Deuze, M. (2004). What is multimedia journalism? Journalism Studies, 5 (2)

Deuze, M. (2005). What is journalism? Professional Identity and ideology of journalist reconsidered. Journalism "Teory, Practice and Criticism, 6(4). 
Elías, C. (2004). La especialización periodística como herramienta para paliar la vulnerabilidad del redactor ante las fuentes manipuladoras. En M. J. Casals (Coord.), Mensajes periodísticos y sociedad del conocimiento. Madrid: Fragua.

FELAFACS (2009). Mapa de los centros y programas de formación de comunicadores y periodistas en América Latina y el Caribe. Lima: Felafacs.

Foro de Periodismo Argentino (2008). Estándares profesionales e indicadores de calidad periodística. Buenos Aires: Universidad de Palermo.

Fuentes N. R. (2014). Postgrados e investigación en Comunicación en México: ¿estancamiento o evolución de la desarticulación múltiple? Comunicación y Sociedad, (22). Recuperado de http://www.revistascientificas.udg.mx/index.php/comsoc/article/view/47/63

Fuller, J. (1997). News values: ideas for an information ages. Chicago: University of Chicago Press.

Gutiérrez Atala, F. (2012). Hacia el nuevo perfil del periodista actual: especializado, negociador, empoderado y autónomo. Revista Iberoamericana de Comunicación, RIC (22).

Gutiérrez-Atala, F., Ferreira-Jiménez, J., \& Pajoni, H. (2015). Estudio sobre los efectos de la presión política y ciudadana en las rutinas profesionales de periodistas de tres ciudades latinoamericanas.Comuni@cción - Revista de Investigación en Comunicación y Desarrollo, 6(2).

Gutiérrez-Atala, F., Odriozola-Chéné, J., Aguirre-Mayorga, C., Bernal-Suárez, J. D., Ferreira, J., Laurencio, L.,Aguirre, P. (2015). Periodismo condicionado: autodiagnóstico sobre cómo cumplen su función los periodistas de Chile, Argentina, Paraguay, Ecuador, Colombia y México en la era de la transparencia. En R. CetinaPresuel, L. Corredoira, \& F. Gutiérrez-Atala (Eds.), Medios y periodistas en la era del Gobierno Abierto y la Transparencia. Madrid: Universidad Complutense de Madrid.

Gutiérrez-Atala, F., Odriozola-Chéné, J., Ferreira, J., Anaya-Ávila, P., \& Pajoni, H. (2016). El peso de la presión social y política: estudio de las limitaciones que condicionan el desempeño de los periodistas en cinco países latinoamericanos. Anuario electrónico de estudios en Comunicación Social « Disertaciones», 9(1), http://doi. org/10.12804/disertaciones.09.01.2016.05

Hanitzsch, T., Anikina, M., Berganza, R., Cangoz, I., Coman, M., Hamada, B., Moreira, S. V. (2010). Modeling perceived influences on journalism: Evidence from a cross-national survey of journalists. Journalism \& Mass Communication Quarterly, 87(1), 5-22. http://doi.org/doi: 10.1177/107769901008700101

Hernández R. M. (2004). La formación universitaria de periodistas en México. 
Comunicación y Sociedad, (1). Recuperado de http://www.revistascientificas.udg.mx/ index.php/comsoc/article/view/4235

Inglehart, R., \& Carballo, M. (1997). Does Latin America exist? (And is there a Confucian culture?): A global analysis of cross-cultural differences. PS: Political Science E Politics, 30(1), http://doi.org/dx.doi.org/10.2307/420668

Kobach, B., y Rosenstiel, T. (2001). "The elements of journalism: what newspeoplesshould know and the public should expect. Nueva York: Three Rivers Press.

López García, X. (2010). La formación de los periodistas en el siglo XXI en Brasil, España, Portugal y Puerto Rico. Revista Latina de Comunicación Social, (65). DOI: 10.4185/RLCS-65-2010-896-231-243

McChesney, R. (2004). The Problem of the Media: U.S. Communication Politics in the Twenty-First Century. New York: MonthlyReviewPress.

Mellado, C. (2009). Periodismo en Latinoamérica: Revisión histórica y propuesta de un modelo de análisis. Comunicar, (33). Recuperado de: http://www.revistacomunicar.com/numeros_anteriores/archivospdf/33/c33-2009-03-011.pdf

Mellado, C. (2010). Reflexiones sobre la oferta académica, la situación laboral y la formación del periodista en Latinoamérica. Revista de Ciencias Sociales (Ve), 16 (1) Recuperado de: http://200.74.222.178/index.php/rcs/article/view/13664/13647

Mellado, C. (2012). The Chilean Jorunalist, en Thle Global Journalist in at 21th Century, D. Weaver \&L. Willnat, Eds. Nueva York: Routledge

Meyer, P. (2004). Saving Journalism: How to nurse the good stuff until it pays, Columbia Journalism Review, Nov-Dec 2004.

Meyer, P. (2004). The Vanishing Newspaper: saving jounalism in te information age. Columbia: University Missouri Press.

Oller-Alonso, M., \& Meier, K. (2012). La cultura periodística de España y Suiza. Madrid: Fragua.

Oller-Alonso, M. (2016). La cultura periodística de América Latina: De dónde viene, dónde está y a dónde... debería ir. Razón y Palabra, 20(93).

Punín, M.I. (2012). Los estudios de comunicación social/periodismo en el Ecuador. Una visión crítica al rol de la universidad y la academia. Razón y Palabra, 17(79).Recuperado de: http://oldversion.razonypalabra.org.mx/N/N79/V79/66_Punin_V79.pdf

Punín, M.I; Rivera, D. \& Cuenca, P. (2014). Cuánto la vocación prima: La situación laboral del periodista digital en Ecuador y su formación académica. ComHumanitas, 5(1). Recuperado de: http://comhumanitas.org/index.php/comhumanitas/article/ view/54/pdf_13 
Ramonet, I. (2011). La explosión del periodismo. Internet pone en jaque a los medios tradicionales. Argentina: Capital Intelectual S.A.

Reese, S. (1999). Hacia una comprensión del periodista global. El modelo de investigación de jerarquía de influencias. Comunicación y Sociedad, 12(2).

Reig, R. (2007). El periodista en la telaraña. Nueva economía, comunicación, periodismo, públicos. Barcelona, España: Anthropos.

Seijas, L (2001). Los sistemas informativos en la era digital. Madrid: Universitas S.A.

Serrano, P. (2012). Periodismo canalla. Los medios contra la información. Barcelona: Icaria.

Servicio Observatorio Internet. (2001). El rostro humano de la cultura general cómo nos afecta Internet. Bogotá, Colombia: Equipo Paulinas.

Shoemaker, P. J., \& Reese, S. D. (2014). Mediating the Message in the 21st Century: A Media Sociology Perspective. New York: Routledge.

Shoemaker, P. \& Reese, S. (1996). Mediating the Message. Theories of Influences on Mass Media Content. New York: Longman. 